\title{
Probiotics for Prophylaxis and Treatment of Urinary Tract Infections in Children
}

\author{
Ilke Beyitler,," and Salih Kavukcu ${ }^{1}$ \\ ${ }^{1}$ Department of Pediatrics, Near East University Hospital, Nicosia, Cyprus \\ "Corresponding author: Ilke Beyitler, Near East University Hospital, Department of Pediatrics, 99138 Nicosia, Cyprus, Mersin 10 Turkey. Tel: +90-05488542670, E-mail: \\ ilkebeyit82@yahoo.com \\ Received 2016 June 28; Accepted 2016 September 30.
}

\begin{abstract}
Context: Urinary tract infection (UTI) is a common and important problem during childhood that may cause chronic morbidities. Live beneficial microorganisms called probiotics have been investigated for their use in the prophylaxis and treatment of UTIs in children.

Evidence Acquisition: We aimed to discuss data about use, mechanisms and effects of probiotics on pediatric UTIs. We searched the literature, including PubMed, Medline and Cochrane databases from January 2001 to May 2016, and limited the search to English language. We used the key words probiotics, urinary tract infection, treatment, prophylaxis and children.

Results: Probiotics inhibit uropathogens by competition for receptors and nutrients, direct killing, immune modulation and production of inhibitory metabolites. There are many organisms that have been used as probiotics. Lactobacillus sp., Bifidobacterium sp. and Saccharomyces boulardii are the most commonly used and investigated probiotics. Although there are various benefits of probiotics for the pediatric population, some reports indicate rare complications such as bacteremia, sepsis, endocarditis, meningitis, UTI, abscesses, fungemia, pneumonia and chorioamnionitis. However, these are much less than the benefits of probiotics yet should be kept in mind. Continuing laboratory and clinical studies are encouraging the use of this strategy for the prevention and treatment of UTI in children.
\end{abstract}

Conclusions: Probiotics can be used as a natural and efficient alternative to antibiotics in UTI prophylaxis and treatment in children.

Keywords: Probiotics, Urinary Tract Infection, Treatment, Prophylaxis, Children

\section{Context}

Urinary tract infection(UTI) is a very common problem in pediatric practice, which may lead to long term health issues such as renal scar, renal failure and hypertension, especially when it is recurrent. Urinary tract infection also increases the healthcare costs of countries. There are various bacteria, viruses, fungi and parasites that cause UTI, but the most frequently seen agent is found to be Escherichia coli (E. coli) (1). Treatment of UTI with antibiotics has led to some side effects, destruction of normal flora and emerging resistant microorganisms are a worldwide difficulty. Nonantibiotic methods have come to be effective against UTI without increasing the resistance rates. Probiotics in this aspect, are beneficial live microorganisms utilized in treatment of several infectious diseases and shown to be effective in UTI prophylaxis and treatment as a current concept. Avoiding antibiotic resistance, restoring a healthy microbiota and supporting the immune system are all exhibited by probiotics (2). In this review, we aimed to discuss current data about the role, mechanisms and effects of probiotics in prophylaxis and treatment of UTIs during childhood.

\section{Evidence Acquisition}

We performed a literature search with PubMed, Medline and Cochrane database from January 2001 to May 2016 to select articles about the use and efficacy of probiotics in prophylaxis and treatment of UTIs in children. We limited our search to literature in the English language. We used the key words probiotics, urinary tract infection, treatment, prophylaxis and children.

\section{Mechanisms Behind Action}

Probiotics have many abilities such as adhesion to cells, avoiding adhesion and aggregation of pathogenic agents and influence the components of flora and activate the immune system. They play a role in the modification of mucosal immunity and enhance other procedures such as production of cytokines, secretion of IgA, phagocytosis and production of inhibitory substances. These substances, which are inhibitory to pathogenic microorganisms are hydrogen peroxide $\left(\mathrm{H}_{2} \mathrm{O}_{2}\right)$, heat stable bacteriocins, antifungal peptides and pH-reducing organic acids like lactic acid and acetic acid. They all have effects

Copyright (C) 2016, Growth \& Development Research Center. This is an open-access article distributed under the terms of the Creative Commons Attribution-NonCommercial 4.0 International License (http://creativecommons.org/licenses/by-nc/4.0/) which permits copy and redistribute the material just in noncommercial usages, provided the original work is properly cited. 
by directly killing pathogens, altering the $\mathrm{pH}$ and receptors. Bacteriocins are antimicrobial peptides that influence other bacteria by formation of pores and inhibition of cell wall synthesis.

Probiotics compete with pathogens for nutrition, they avoid the attachment of pathogens and activity of toxins produced by microorganisms. They increase the proliferation of mucosa, which results in better barrier defence. Probiotics also modify innate and adaptive immune systems and avoid translocation of bacterial pathogens. Another mechanism of probiotics against uropathogens is activation of NF-kappa- $\beta$ and Tumor Necrosis Factor- $\alpha$ (TNF$\alpha$ ) in bladder cells (3-5).

Some in vitro studies demonstrated that lactobacilli produce mucin, biosurfactant barrier and bacteriocin. They compete for receptors on uroepithelium thus avoiding the adhesion of pathogens. They have been shown to decrease inflammatory cytokines such as IL-8 and COX or expression of virulence factors. They also increase immune system response via IgA, IL-10 and IL-12.

Lactobacillus acidophilus (L. acidophilus) has been shown to inhibit $E$. coli in vitro by prevention of adhesion and producing $\mathrm{H}_{2} \mathrm{O}_{2}$ and biosurfactant. Lactobacillus rhamnosus GR-1 attached to uroepithelium and avoided the growth and adhesion of uropathogens. Lactobacillus reuteri RC-14 avoided uropathogen adhesions with $\mathrm{H}_{2} \mathrm{O}_{2}$ and biosurfactant. Lactobacillus casei in the bladder of animals was proved to be very successful in the eradication of $84 \%$ of the uropathogens by stimulating immune response and natural killer cells (6).

Another probiotic, which contains E. coli Nissle 1917 was analyzed and shown to be useful for prevention of pediatric UTIs by its microcin effect and rapid growth rate as the uropathogens mostly arrive from the gut (7).

Another study found that lactic acid, $\mathrm{H}_{2} \mathrm{O}_{2}$ and Lactobacillus in culture supernatants inhibited growth, increased promoter activity of outer membrane proteins of the host and downregulated type 1 and P fimbriae of E. coli that have a role in attachment of uropathogens (8).

Downregulation of proinflammatory cytokines such as Interleukin (IL)-6, IL-8, IL-10, IL-12 and TNF- $\alpha$ was possible with $L$. rhamnosus GR-1 and L. reuteri RC-14. Antiinflammatory results were observed both in serum and urine samples of the patients. The UTI symptoms can also be managed easily as a result of the downregulation of these cytokines (9).

Consumption of L. gasseri and L. coryniformis increased natural killer cells and IgA concentrations of the host (10).

Immunomodulatory proteins produced by L. rhamnosus GR-1 were found to strengthen the activity of nuclear factor-kappa B (NF- $\kappa \mathrm{B})$ in E. coli-stimulated bladder cells. Nuclear factor- $\kappa \mathrm{B}$ is a transcription factor that regulates immunological genes. This method has been shown to enhance the clearance of uropathogens and used for prevention and treatment of UTI, which may also be valuable if live bacteria are not preferred to be used (11).

In vitro activity of commercial Lactocabilli may also help maintain a balanced urogenital flora, displace uropathogenic E. coli (UPEC) and reduce UTI rates. They upregulated antimicrobials, inhibited growth with acids and reduced survival of pathogens. Lactic acid was measured at high amounts, and reduced $\mathrm{pH}$, permeabilized outer membranes of bacteria, had chelating effects and inhibited growth by capturing nutrients such as iron (12).

\section{Trials on the Current Concept}

Probiotics have been used in observational studies in several countries for 20 years and found to be safe and efficient in thousands of adults and infants (2).

In a randomized, double-blind placebo controlled study, oral intake of probiotics containing Lactobacillus rhamnosus GR-1 and L. reuteri RC-14 were shown to reduce pathogen load in the urogenital tract (13).

A prospective randomized controlled trial, with 120 children having persistent Vesicoureteral Reflux (VUR) for one year given L. acidophilus $\left(2 \times 10^{8} \mathrm{CFU} /\right.$ day $)$ or low dose trimethoprim/sulfamethoxazole prophylaxis and probiotics, found that these combinations were as effective as antibiotic prophylaxis and much more effective than a previous report including children with no prophylaxis (6).

Breastfeeding as a natural probiotic has been shown to prevent UTI in 200 infants in a case controlled study. As duration of breastfeeding increased, risk of UTI decreased significantly (14).

Urinary Tract Infection rates were found to be less frequent in a double-blind study with 585 preterm infants given Lactobacillus GG compared to the placebo group in neonatal intensive care unit (15).

There are also other reports of efficient prophylaxis with probiotics such as L. acidophilus capsules in pediatric recurrent UTIs (16).

In a small study on ten adult females with recurrent UTI, probiotic preparation containing $10^{9} \mathrm{CFU}$ of L. rhamnosus GR-1 and L. fermentum RC-14 was given orally to the patients twice daily for two weeks. Infection parameters resolved one week after this treatment in six of the cases (17).

In a double-blind multicentre trial including 252 postmenopausal females with recurrent UTIs, oral prophylaxis was given either with trimethoprim / sulfamethoxazole or $10^{9} \mathrm{CFU}$ of L. rhamnosus GR-1 and L. reuteri RC-14 two times a day, for one year. Probiotic and antibiotic prophylaxis were found similarly successful in reducing UTI recurrence, yet 
the group receiving probiotic had the advantage of not experiencing resistance (18).

Lactobacillus rhamnosus GR-1 and L. reuteri RC-14 were also shown to protect the urogenital tract from UPEC colonization and infection on experimental cultures (8).

In a preliminary randomized controlled trial with 85 children aged 3 to 15 years, who had recurrent UTI and unilateral VUR, one group was given prophylactic nitrofurantoin and the second group was given nitrofurantoin and probiotic (L. acidophilus and Bifidobacterium lactis $10^{7}$ $\mathrm{CFU} / \mathrm{mL}$ ) together for a certain period intermittently during more than a year. Follow up continued for two to three years and both groups had similarly decreased incidence of UTI. However, nitrofurantoin together with probiotics was found to be more effective in reducing febrile UTIs (19).

Among 600 samples of urine infected with $>10,000$ $\mathrm{CFU} / \mathrm{mL}$ multidrug resistant bacteria, Lactobacilli probiotics (L. acidophilus, L. casei and L. rhamnosus) did not have antagonistic effect on tests with Enterococcus, Enterobacter or Klebsiella pneumoniae. However, probiotics had an inhibitory effect on E. coli. Moreover, L. casei was the most effective bacteria (20).

Three hundred and forty-four children aged three months to 12 years receiving broad-spectrum antibiotics in PICU were administered a probiotic mixture containing L. acidophilus, L. rhamnosus, Bifidobacterium bifidum, B. longum, Saccharomyces boulardii and Streptococcus thermophilus for seven days. Prevalence of candidemia and candiduria was successfully reduced with probiotics compared to the control group of 376 children not taking probiotics (21).

Overall, $5 \times 10^{9} \mathrm{CFU}$ of $S$. boulardii either in capsule or powder form was administered orally to 24 children aged 3 to 16 years for five days. Number of $E$. coli colonies in colon was measured before and after treatment. In children's analyzed stool samples, E. coli colonization was found to be decreased. Therefore, prevention of UTIs can be possible using S. Boulardii (22).

In vitro effect of single and multi-strain probiotics on E. coli and Enterococcus faecalis as urinary tract pathogens was tested. The probiotic mixtures used were two lactobacilli (L. acidophilus and L. plantarum), three lactobacilli ( $L$. acidophilus, L. fermentum and L. rhamnosus), four lactobacilli (L. acidophilus, L. fermentum, L. rhamnosus and L. plantarum) and a commercial mixture. It consisted of L. acidophilus, $L$. delbrueckiibulgaricus, L. casei, L. plantarum, L. rhamnosus, L. salivarius, L. helveticus, L. lactis, B. bifidum, B. breve, B. infantis, $B$. longum, S. thermophilus and Bacillus subtilis. Probiotics as single and in combination forms were equally effective on preventing uropathogen growth and reducing UTI risk (5).

A retrospective study compared the effectivity of prophylaxis with probiotics (L. acidophilus $1 \times 10^{8} \mathrm{CFU} / \mathrm{g}$ or
L. acidophilus + L. rhamnosus $2 \times 10^{9} \mathrm{CFU} / \mathrm{g}$ ), antibiotics (trimethoprim/sulfamethoxazole) and no-prophylaxis in three groups. There was a total of 191 infants, who had acute pyelonephritis and anatomically normal urinary tracts. Incidence of UTI for six months after pyelonephritis was investigated. Probiotics were significantly more effective for prophylaxis than no-prophylaxis. Although not statistically significant, probiotics also resulted in lower UTI rates than the antibiotic group. The resistance rates of $E$. coli were much lower in the probiotic group than in the other two groups (23).

However, there are some rare reports that probiotics may lead to serious infections both in immunocompetent and immunocompromised patients and the detection of causative agent is difficult as they are usually regarded as contaminants. An adult, who had diverticulosis and hemorrhoidal bleeding was reported to have $L$. casei bacteremia treated successfully with antibiotics (24).

One term newborn with multiple congenital anomalies and one extremely preterm newborn had sepsis due to $5 \times 10^{9} \mathrm{CFU}$ oral L. rhamnosus GG supplementation given to avoid antibiotic associated compications or necrotizing enterocolitis. There are also other adult or pediatric cases of bacteremia, sepsis, fungemia, infectious endocarditis, pneumonia, chorioamnionitis, meningitis, UTI and abscesses due to probiotics like lactobacillus, bifidobacterium and saccharomyces. The majority of these case reports have a serious or chronic disease such as gastrointestinal pathology, prematurity, diabetes, heart defect, cerebral palsy, burn, recent surgery or immunosuppression due to several reasons. However, these should not discourage the use of probiotics as the number of these cases are much lower than the great population that benefits from probiotics. The important point is that clinicians should keep in mind that these agents may cause serious infections especially in certain patient groups, who have underlying health problems (25-27).

\section{Recommended Dosages and Duration}

There is a wide range of dosage and duration choices regarding probiotic administration depending on patient's age, clinical status and clinician's perspective. The dose of Lactobacillus rhamnosus was reported to range from $3 \times 10^{9} \mathrm{CFU}, 5 \times 10^{9} \mathrm{CFU}$ to $10 \times 10^{9} \mathrm{CFU}$ and duration of supplemantation differed from 4 to 169 days. Other trials recommended the dosage of Lactobacillus species and Bifidobacterium species ranging from $1 \times 10^{9} \mathrm{CFU}$ in children to $35 \times 10^{9} \mathrm{CFU}$ in adults depending on preparation and duration of up to six weeks. Saccharomyces boulardii dose has been recommended as $250 \mathrm{mg}$ to $500 \mathrm{mg}(4,28)$. 


\section{Conclusion}

Efficacy and safety of probiotics for UTIs have been investigated and shown implicating results. Probiotics may be promising and effective natural immunomodulators for prevention and treatment of UTIs in children. There is sufficient data to suggest that this approach is a valuable choice that should be encouraged regarding its utilization in UTIs and benefits from all of the advantages simultaneously.

\section{Acknowledgments}

The authors had no financial support or financial interest.

\section{Footnotes}

Authors' Contribution: Study concept and design, Ilke Beyitler and Salih Kavukcu; acquisition of data, Ilke Beyitler; analysis and interpretation of data, Ilke Beyitler and Salih Kavukcu; drafting of the manuscript, Ilke Beyitler; critical revision of the manuscript for important intellectual content, Salih Kavukcu; administrative, technical and material support, Salih Kavukcu; Study supervision, Salih Kavukcu.

Funding/Support: The authors had no financial support or financial interest.

\section{References}

1. Madden-Fuentes RJ, Arshad M, Ross SS, Seed PC. Efficacy of Fluoroquinolone/Probiotic Combination Therapy for Recurrent Urinary Tract Infection in Children: A Retrospective Analysis. Clin Ther. 2015;37(9):2143-7. doi: 10.1016/j.clinthera.2015.06.018. [PubMed: 26233470].

2. Borchert D, Sheridan L, Papatsoris A, Faruquz Z, Barua JM, Junaid I, et al. Prevention and treatment of urinary tract infection with probiotics: Review and research perspective. Indian J Urol. 2008;24(2):13944. [PubMed: 19468386].

3. Amdekar S, Singh V, Singh DD. Probiotic therapy: immunomodulating approach toward urinary tract infection. Curr Microbiol. 2011;63(5):484-90. doi: 10.1007/s00284-011-0006-2. [PubMed: 21901556].

4. Singhi SC, Kumar S. Probiotics in critically ill children. F1000Research. 2016;5:407. doi: 10.12688/f1000research.7630.1.

5. Chapman CM, Gibson GR, Rowland I. Effects of single- and multistrain probiotics on biofilm formation and in vitro adhesion to bladder cells by urinary tract pathogens. Anaerobe. 2014;27:71-6. doi: 10.1016/j.anaerobe.2014.02.001. [PubMed: 24583095].

6. Lee SJ, Shim YH, Cho SJ, Lee JW. Probiotics prophylaxis in children with persistent primary vesicoureteral reflux. Pediatr Nephrol. 2007;22(9):1315-20. doi: 10.1007/s00467-007-0507-1. [PubMed: 17530295].

7. Storm DW, Patel AS, Koff SA, Justice SS. Novel management of urinary tract infections. Curr Opin Urol. 2011;21(4):328-33. doi: 10.1097/MOU.ob013e328346d4ee. [PubMed: 21519273].
8. Cadieux PA, Burton J, Devillard E, Reid G. Lactobacillus by-products in hibit the growth and virulence of uropathogenic Escherichia coli. Physiol Pharmacol. 2009;60 Suppl 6:13-8. [PubMed: 20224146].

9. Anukam KC, Hayes K, Summers K, Reid G. ProbioticLactobacillus rhamnosusGR-1 andLactobacillus reuteriRC-14 May Help Downregulate TNF-Alpha, IL-6, IL-8, IL-10 and IL-12 (p70) in the Neurogenic Bladder of Spinal Cord Injured Patient with Urinary Tract Infections: A Two-Case Study. Adv Urol. 2009;2009:1-5. doi: 10.1155/2009/680363.

10. Olivares M, Diaz-Ropero MP, Gomez N, Lara-Villoslada F, Sierra S, Maldonado JA, et al. The consumption of two new probiotic strains, Lactobacillus gasseri CECT 5714 and Lactobacillus coryniformis CECT 5711, boosts the immune system of healthy humans. Int Microbiol. 2006;9(1):47-52. [PubMed:16636989].

11. Karlsson M, Scherbak N, Khalaf H, Olsson PE, Jass J. Substances released from probiotic Lactobacillus rhamnosus GR-1 potentiate NFkappaB activity in Escherichia coli-stimulated urinary bladder cells. FEMS Immunol Med Microbiol. 2012;66(2):147-56. doi: 10.1111/j.1574695X.2012.00994.x. [PubMed: 22620976]

12. Delley M, Bruttin A, Richard M, Affolter M, Rezzonico E, Brück WM, et al. In vitroactivity of commercial probioticLactobacillusstrains against uropathogenicEscherichia coli. FEMS Microbiology Letters. 2015;362(13):96. doi:10.1093/femsle/fnv096.

13. Anukam K, Osazuwa E, Ahonkhai I, Ngwu M, Osemene G, Bruce AW, et al. Augmentation of antimicrobial metronidazole therapy of bacterial vaginosis with oral probiotic Lactobacillus rhamnosus GR-1 and Lactobacillus reuteri RC-14: randomized, doubleblind, placebo controlled trial. Microbes Infect. 2006;8(6):1450-4. doi: 10.1016/j.micinf.2006.01.003. [PubMed:16697231].

14. Marild S, Hansson S, Jodal U, Oden A, Svedberg K. Protective ef fect of breastfeeding against urinary tract infection. Acta Paediatr. 2004;93(2):164-8. [PubMed: 15046267].

15. Dani C, Biadaioli R, Bertini G, Martelli E, Rubaltelli FF. Probiotics feeding in prevention of urinary tract infection, bacterial sepsis and necrotizing enterocolitis in preterm infants. A prospective doubleblind study. Biol Neonate. 2002;82(2):103-8. [PubMed: 12169832].

16. Gerasimov SV. Probiotic Prophylaxis in Pediatric Recurrent Urinary Tract Infections. Clin Ped. 2004;43(1):95-8. doi: 10.1177/000992280404300113.

17. Reid G, Bruce AW, Fraser N, Heinemann C, Owen J, Henning B. Oral probiotics can resolve urogenital infections. FEMS Immunol Med Microbiol. 2001;30(1):49-52. [PubMed: 11172991].

18. Beerepoot MA, ter Riet G, Nys S, van der Wal WM, de Borgie CA de Reijke TM, et al. Lactobacilli vs antibiotics to prevent urinary tract infections: a randomized, double-blind, noninferiority trial in postmenopausal women. Arch Intern Med. 2012;172(9):704-12. doi: 10.1001/archinternmed.2012.777. [PubMed: 22782199].

19. Mohseni MJ, Aryan Z, Emamzadeh-Fard S, Paydary K, Mofid V, Joudaki $\mathrm{H}$, et al. Combination of probiotics and antibiotics in the prevention of recurrent urinary tract infection in children. Iran J Pediatr 2013;23(4):430-8. [PubMed: 24427497].

20. Naderi A, Kasra-Kermanshahi R, Gharavi S, Imani Fooladi AA, Abdollahpour Alitappeh M, Saffarian P. Study of antagonistic effects of Lactobacillus strains as probiotics on multi drug resistant (MDR) bacteria isolated from urinary tract infections (UTIs). Iran J Basic Med Sci. 2014;17(3):201-8. [PubMed: 24847423].

21. Kumar S, Singhi S, Chakrabarti A, Bansal A, Jayashree M. Probiotic use and prevalence of candidemia and candiduria in a PICU. Pediatr Crit Care Med. 2013;14(9):409-15. doi: 10.1097/PCC.0b013e31829f5d88. [PubMed: 23965638].

22. Akil I, Yilmaz O, Kurutepe S, Degerli K, Kavukcu S. Influence of oral intake of Saccharomyces boulardii on Escherichia coli in enteric flora. Pediatr Nephrol. 2006;21(6):807-10. doi: 10.1007/s00467-006-0088-4. [PubMed: 16703374].

23. Lee SJ, Cha J, Lee JW. Probiotics prophylaxis in pyelonephritis infants with normal urinary tracts. World Journal of Pediatrics. 2016 doi: 10.1007/s12519-016-0013-2. 
24. Tommasi C, Equitani F, Masala M, Ballardini M, Favaro M, Meledandri $\mathrm{M}$, et al. Diagnostic difficulties of Lactobacillus casei bacteraemia in immunocompetent patients: a case report.JMed Case Rep. 2008;2:315 doi: 10.1186/1752-1947-2-315. [PubMed: 18826603].

25. Dani C, Coviello CC, Corsini II, Arena F, Antonelli A, Rossolini GM. Lactobacillus Sepsis and Probiotic Therapy in Newborns: Two New Cases and Literature Review. AJP Rep. 2016;6(1):25-9. doi: 10.1055/s-00351566312. [PubMed: 26929865].

26. Zbinden A, Zbinden R, Berger C, Arlettaz R. Case series of Bifi- dobacterium longum bacteremia in three preterm infants on probiotic therapy. Neonatology. 2015;107(1):56-9. doi: 10.1159/000367985. [PubMed: 25402825].

27. Stefanatou E, Kompoti M, Paridou A, Koutsodimitropoulos I, Giannopoulou P, Markou N, et al. Probiotic sepsis due to Saccharomyces fungaemia in a critically ill burn patient. Mycoses. 2011;54(5):643-6. doi: 10.1111/j.1439-0507.2010.01963.x. [PubMed: 21672040].

28. Kligler B, Cohrssen A. Probiotics. Am Fam Physician. 2008;78(9):1073-8. [PubMed: 19007054]. 